\title{
GaN:ZnO Solid Solution as a Photocatalyst for Visible-Light-Driven Overall Water Splitting
}

Kazuhiko Maeda ${ }^{+}$, Tsuyoshi Takata ${ }^{+}$, Michikazu Hara ${ }^{\ddagger}$, Nobuo Saito ${ }^{\dagger}$, Yasunobu Inoue ${ }^{\dagger}$, Hisayoshi Kobayashi", and Kazunari Domen ${ }^{+*}$

Department of Chemical System Engineering, The University of Tokyo, 7-3-1 Hongo, Bunkyo-ku, Tokyo 113-8656, Japan, Chemical Resources Laboratory, Tokyo Institute of Technology, 4259 Nagatsuta, Midori-ku, Yokohama 226-8503,

Japan, Department of Chemistry, Nagaoka University of Technology, Nagaoka 940-2188, Japan, and Department of Chemistry and Materials Technology, Faculty of Engineering and Design, Kyoto Institute of Technology,

Goshokaido-cho, Matsugasaki, Sakyo-ku, Kyoto 606-8585, Japan

\section{Supporting Information}

\section{Full Citations in Ref. 8:}

Sato, J.; Saito, N.; Yamada, Y.; Maeda, K.; Takata, T.; Kondo, J. N.; Hara, M.; Kobayashi, H.; Domen, K.; Inoue, Y. J. Am. Chem. Soc. 2005, 127, 4150-4151.

\section{Full Citations in Ref. 14:}

Tsukazaki, A.; Ohtomo, A.; Onuma, T.; Ohtani, M.; Makino, T.; Sumiya, M.; Ohtani, K.; Chichibu, S. F.; Fuke, S.; Segawa, Y.; Ohno, H.; Koinuma, H.; Kawasaki, M. Nature Mater. 2005, 4, 42-46. 